\title{
Extensive Numerical Results for Integrable Case of Standard Map
}

\author{
Ugur Tirnakli* \\ Department of Physics, Faculty of Science, Ege University, 35100 Izmir, TURKEY
}

Constantino Tsallis ${ }^{\dagger}$

Centro Brasileiro de Pesquisas Fisicas and National Institute of Science and Technology for Complex Systems, Rua Xavier Sigaud 150, Rio de Janeiro 22290-180, BRAZIL

(Received 24 November, 2019)

\begin{abstract}
In recent years, conservative dynamical systems have become a vivid area of research from the statistical mechanical characterization viewpoint. With this respect, several areapreserving maps have been studied. It has been numerically shown that the probability distribution of the sum of the suitable random variable of these systems can be well approximated by a Gaussian ( $q$-Gaussian) when the initial conditions are randomly selected from the chaotic sea (region of stability islands) in the available phase space. In this study, we will summarize these results and discuss a special case for the standard map, a paradigmatic example of area-preserving maps, for which the map is totally integrable.
\end{abstract}

PACS numbers: 05.20.-y, 05.10.-a, 05.45.-a

Keywords: dynamical systems, conservative maps, central limit theorem

DOI: https://doi.org/10.33581/1561-4085-2020-23-2-149-152

In the area of dynamical systems, area-preserving conservative low-dimensional mappings are very important $[1,2]$ and constitute an extremely vivid research field. Among many others, the most well-known paradigmatic example of such mappings is the so-called Taylor-Chirikov or standard map defined as

$$
\begin{aligned}
& p_{n+1}=p_{n}-K \sin \left(x_{n}\right), \\
& x_{n+1}=x_{n}+p_{n+1},
\end{aligned}
$$

where $p$ and $x$ are taken as modulo $2 \pi$ and $K$ is the map parameter which controls the amount of nonlinearity and nonintegrability of the system. Typical examples of the phase space are given in Figure 1. It is evident from this figure that the case $K=0$ and $K=8$ are extreme cases in the sense that the phase space consists of only stability islands for the former, whereas it only exhibits chaotic sea in the latter. The other two

\footnotetext{
*E-mail: ugur.tirnakli@ege.edu.tr

${ }^{\dagger}$ E-mail: tsallis@cbpf.br; Also at Complexity Science Hub Vienna, Josefstädter Strasse 39, 1080 Vienna, AUSTRIA; Also at Santa Fe Institute, 1399 Hyde Park Road, Santa Fe, New Mexico 87501, USA
}

cases demonstrate how the chaotic sea develops as $K$ increases from 0 to larger values. This map was firstly introduced by Taylor [3] and Chirikov [4], and was afterwards shown to mimic many dynamical features of real physical systems $[5,6]$. Besides the dynamical properties that have been extensively studied in the literature, the statistical mechanical characterization of the map has recently been discussed in details [7, 8]. To achieve this, one needs to define a random variable from the map variable $x$ as

$$
y=\sum_{n=1}^{T}\left(x_{n}-\langle x\rangle\right)
$$

where $\langle\cdots\rangle$ implies averaging over a large number of iterations $T$ and a large number of randomly chosen initial conditions $M$, more precisely,

$$
\langle x\rangle=\frac{1}{M} \frac{1}{T} \sum_{j=1}^{M} \sum_{n=1}^{T} x_{n}^{(j)} .
$$

For arbitrary values of the control parameter $K$, the probability distribution of these sums 
appears to be modelled remarkably well by $q$ Gaussians defined as

$$
P_{q}(y ; 0 ; 1)=A_{q} \sqrt{B_{q}} \exp _{q}\left(-y^{2}\right),
$$

where $A_{q}$ is the normalization factor, $B_{q}$ is a parameter which characterizes the width of the distribution and $\exp _{q}(x)$ is called $q$-exponential which is given by

$$
\exp _{q}(x)=\left[1+(1-q) B_{q} x\right]^{\frac{1}{1-q}} .
$$

It is worth to note here that the Gaussian is a special case of Equation 4 in the $q \rightarrow 1$ limit. Very similar to the fact that the Gaussians are the attractors of the standard central limit theorem, the $q$-Gaussians are the attractors of the generalized central limit theorem [9-11]. It is numerically shown that, if the initial conditions are taken randomly from the initial conditions that are inside the stability islands, this distribution is a $q$-Gaussian with $q \simeq 1.935$, whereas it is a Gaussian if the initial conditions are taken from the chaotic sea. The reason for this kind of behavior can be discussed by considering the Lyapunov spectrum for each initial condition without making an average over all initial conditions as usually done in the literature. Typical examples of such spectra, calculated by using Benettin algorithm [12], are given in Figure 2 for the same map parameter values used in Figure 1. It is clearly seen that, all initial conditions from the chaotic portion of the phase space have positive Lyapunov exponents, sensibly larger than zero, whereas they are always in the vicinity of zero if the initial conditions are from the stability islands. The ergodic nature of the trajectories living in the chaotic sea gives rise to a Gaussian probability distribution as expected. On the other hand, ergodicity breakdown for the trajectories of stability islands with nearly zero Lyapunov exponents causes non-Gaussian probability distribution, which appear to sensibly approach a $q$-Gaussian with $q \simeq 1.935$.
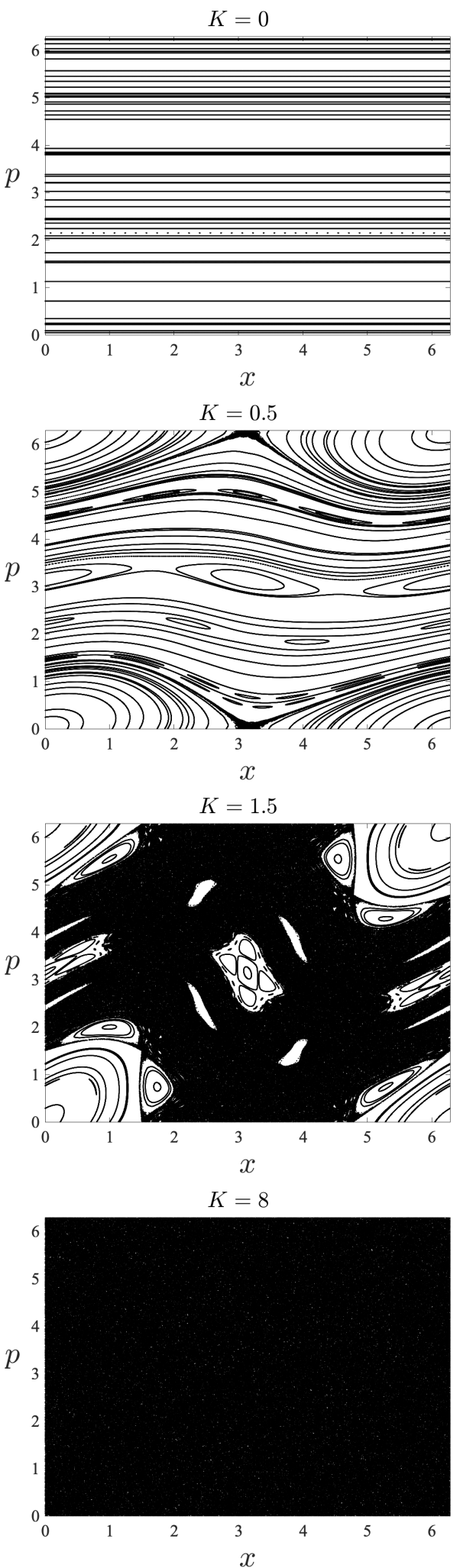

FIG. 1. Phase space portrait of the standard map with some representative values of map parameter $K$. 

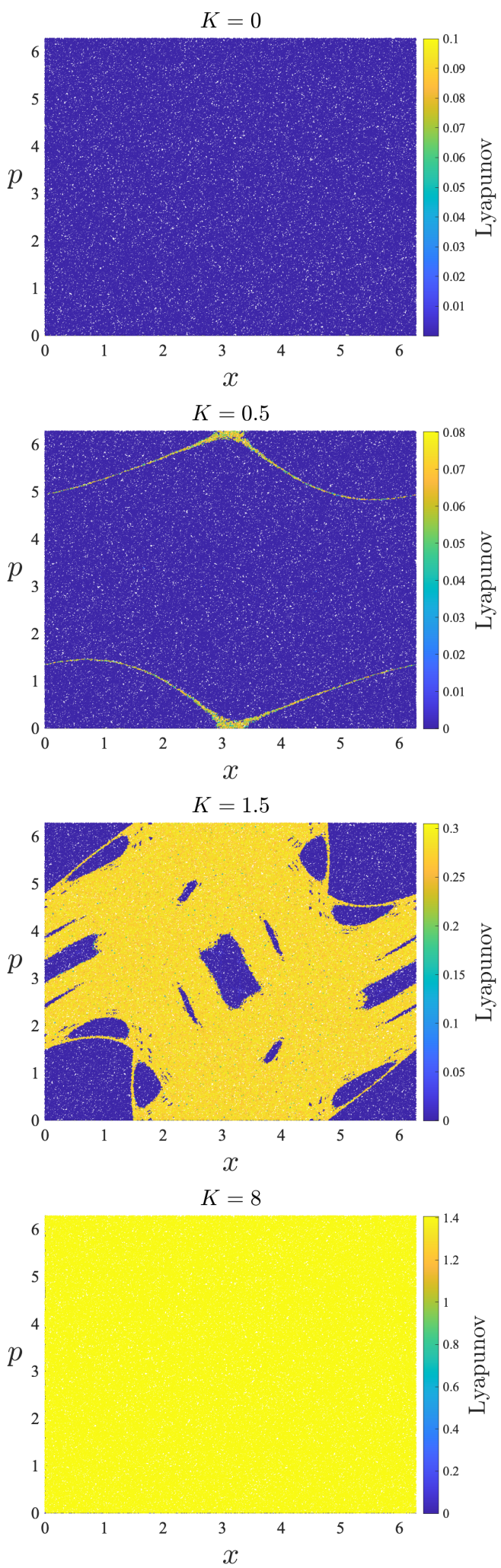

FIG. 2. (color online) Lyapunov spectrum for the standard map for the same representative values of $K$ indicated in Figure 1.

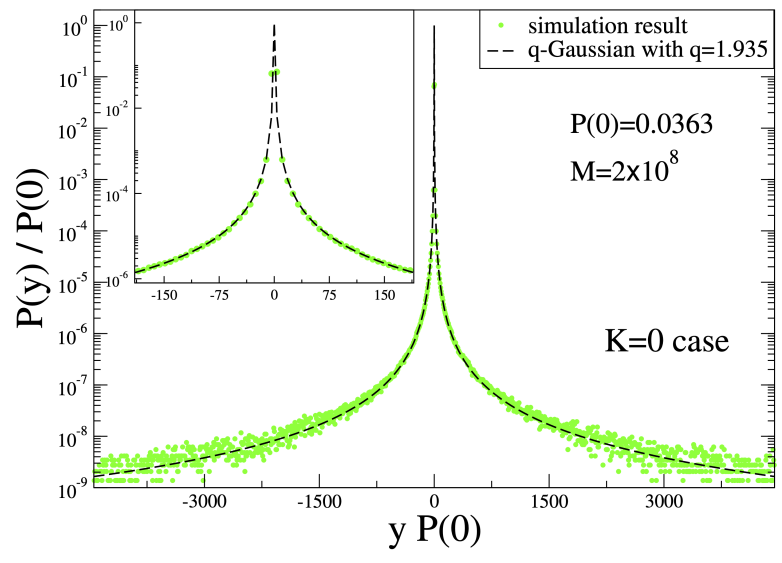

FIG. 3. (color online) Probability distribution obtained for the $K=0$ case. A quite large number of initial conditions is used $\left(M=2 \times 10^{8}\right)$ for good statistics. The number of summands used in the simulations is also large enough $\left(T=2^{22}\right)$ in oder to visualize the limit distribution. The precise method for the determination of $P(0)$ has been introduced in [13].

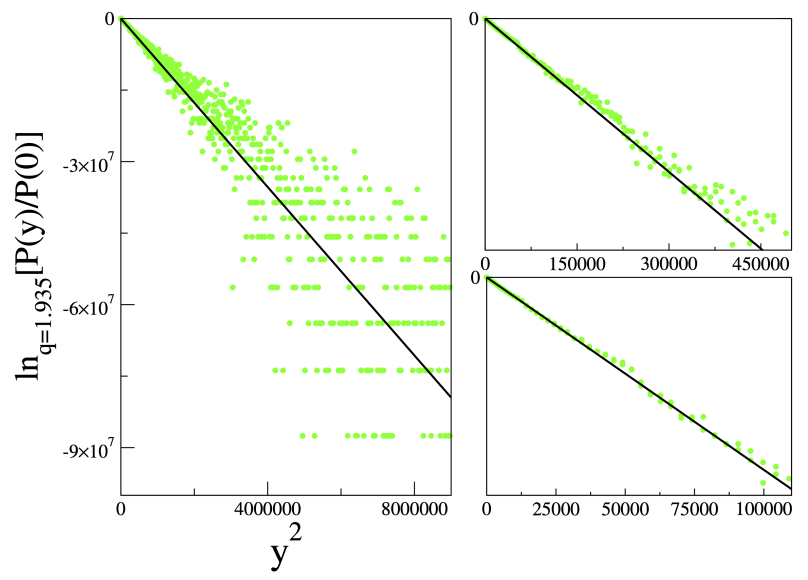

FIG. 4. (color online) $q$-logarithmic representation of the probability distribution obtained for the $K=0$ case. The black straight lines precisely represent the $q$-Gaussian distribution in different scales.

For the calculation of the limit probability distribution with a given map parameter value, one needs to randomly choose a large number of initial conditions (we use $M=2 \times 10^{8}$ ) from the entire phase space and use a large enough number of iteration steps (we use $T=2^{22}$ ) in order to obtain a satisfactory statistical description of the system. Normally one can plot the probability 
distribution $P(y)$ with respect to the random variable $y$, but another way of representing the same plot is to scale the axis by $P(0)$ as done in Figure 3 for $K=0$ case. In this way, one can guarantee that the widths of the distributions will always be the same and can be calculated from the normalization of the probability distribution. Moreover, this scaling provides one more bonus in the sense that now the only fitting parameter that remains in Equation 4 is the value for $q$. We also represent the same data in $q$-logarithmic plot in Figure 4. Here, $q$-logarithm is the inverse function of the $q$-exponential given in Equation 5 and defined as

$$
\ln _{q}(x)=\frac{x^{1-q}-1}{1-q} .
$$

Since the standard map is only integrable for $K=$ 0 , this case is not only more interesting than the others but also a better candidate for attempting analytical treatment. Strictly speaking, when $K=0$, the map becomes

$$
x_{n+1}=x_{n}+n p_{0} \quad \text { with } \quad p_{0}=\text { constant },
$$

which is obviously far simpler than the general standard map with $K \neq 0$. It is certainly intriguing to verify that the limit distribution of this integrable case is very well approximated by a $q$-Gaussian with $q \simeq 1.935$. This observation appears to deserve intensive work, either analytic and/or numeric, to better understand the reasons and underlying dynamics triggering this simple tendency, which so well exhibits $q$-statistics.

\section{Acknowledgments}

We warmly thank stimulating related conversations with J. J. P. Veerman, A. Bountis, C. G. Antonopoulos, and C. Beck. This work has been partially supported by TUBITAK (Turkish Agency) under the Research Project number 115F492, and by CNPq and Faperj (Brazilian Agencies).

\section{References}

[1] R.C. Hilborn. Chaos and nonlinear dynamics: An introduction for scientists and engineers. (Oxford University Press, New York, 2000).

[2] G.M. Zaslavsky. Hamiltonian Chaos and Fractional Dynamics. (Oxford University Press, New York, 2005).

[3] J.B. Taylor, Culham Lab. Prog. Report CLMPR-12 (1969).

[4] B.V. Chirikov. Phys. Rep. 52, 263 (1979).

[5] R.Z. Sagdeev, D.A. Usikov, G. Zaslavsky. Nonlinear Physics: From the Pendulum to Turbulence and Chaos. (Harwood Acad. Publ., New York, 1988).

[6] E. Ott. Chaos in Dynamical Systems.
(Cambridge University Press, Cambridge, 1993).

[7] U. Tirnakli, E. P. Borges. Sci. Rep. 6, 23644 (2016).

[8] G. Ruiz et al. J. Stat. Mech. 063403 (2017).

[9] S. Umarov, C. Tsallis, S. Steinberg. Milan J. Math. 76, 307 (2008).

[10] S. Umarov et al. J. Math. Phys. 51, 033502 (2010).

[11] M.G. Hahn, X.X. Jiang, S. Umarov. J. Phys. A. 43, 165208 (2010).

[12] G. Benettin et al. Phys. Lett. A. 118, 325 (1986).

[13] U. Tirnakli and C. Beck, in preparation. 